\title{
Modeling the Acquisition Front-End in High Resolution Gamma-Ray Imaging
}

\author{
Pedro Guerra, Student Member, IEEE, Juan E. Ortuño, Juan José Vaquero, Senior Member, IEEE,
} George Kontaxakis, Senior Member, IEEE, Manuel Desco, and Andrés Santos, Senior Member, IEEE

\begin{abstract}
The availability of synthetic realistic data eases design optimization, algorithm evaluation and verification of any digital system where a significant amount of digital signal processing is performed. The evolution of positron emission tomography cameras towards continuous sampling of individual position-sensitive photomultiplier anodes with processing algorithms implemented on digital programmable logic devices creates a new framework where new approaches to the $\gamma$-event detection are possible. We have developed a system model of the acquisition chain, including multi-layer phoswich, photomultiplier, front-end analog electronics, data acquisition and digital processing. This processing includes estimation algorithms for the most relevant event parameters: energy, layer-of-interaction, time picking-off and event location. The selected simulation platform couples gently to digital hardware simulation tools, in such a way that implemented models may generate real-like stimuli for the digital system under development. The modeling of the whole front-end electronics enables deeper understanding and tuning of different system trade-offs and provides a rapid and soft transition between specification and hardware development.
\end{abstract}

Index Terms-Continuous sampling, depth of interaction, front-end electronics, hardware validation, nuclear pulse processing, positron emission tomography (PET) modeling.

\section{INTRODUCTION}

$\mathbf{I}$ MAGE quality in positron emission tomography (PET) is strongly influenced by the gamma detector capability to estimate energy, timing and positioning of the $\gamma$-event. Traditionally gamma rays are detected by scintillator crystals, which absorb part of the gamma energy, producing a scintillated light pulse. These optical pulses are then amplified, commonly by a position-sensitive photomultiplier tube (PS-PMT), and readout via charge division techniques [1], [2]. Recent experimental results have shown that processing of individual PS-PMT channels might provide better position estimation and image quality, at the cost of higher complexity and more supporting electronics [3]. Spatial resolution and resolution uniformity can be further improved reducing the parallax error by means of considering a multi-layer detector [4], also known as phoswich.

Manuscript received November 10, 2004; revised April 4, 2006. This work has been funded by the Spanish Ministry of Culture and Science (MEC) through the FPU grant program as well as by the IM3 Thematic Network (G03/185) and the research project TEC2004-07052-C02-02.

P. Guerra, J. E. Ortuño, G. Kontaxakis, and A. Santos are with the Electronic Engineering Department, Universidad Politécnica, Madrid, Spain E-20040 (e-mail: pguerra@die.upm.es; e-mail: juanen@die.upm.es; e-mail: gkont@die.upm.es; andres@die.upm.es).

J. J. Vaquero and M. Desco are with the Hospital General Universitario Gregorio Marañón, Madrid, Spain E-28007 (e-mail: juanjo@mce.hggm.es; desco@mce.hggm.es).

Digital Object Identifier 10.1109/TNS.2006.875065
This work focuses on modeling both the system front-end electronics as well as the digital processing algorithms. This approach eases system internal characteristics understanding, providing a transition between system specification and hardware development, and will facilitate the optimization of the design of future PET detector modules [5].

The system to be modeled consists of a multi-layer detector coupled to a PS-PMT, whose anode outputs are further processed and read by free running analog-to-digital converters (ADCs) at a given acquisition frequency $f_{s}$. Dedicated digital logic processes and characterizes the detected pulses. The motivation to investigate this architecture is the higher spatial resolution and uniformity resolution that can be achieved when the system is able to identify the depth-of-interaction (DOI) [6] in real time, combined with the potential benefits of performing digital processing on each individual channel to increase signal-to-noise ratio and improve position estimation, via local energy integration. Such scheme may also improve timing resolution by means of correcting transit time differences among channels before energy integration and pulse processing.

The presented models provide the framework to simulate the complete acquisition chain. On one hand a functional simulator produces the waveforms that will be generated by the analog readout electronics and sampled by the ADCs, on the other hand a digital hardware simulator takes this sampled data as input stimuli for the detection algorithms. This cosimulation approach provides the mechanisms to validate and characterized the implemented digital algorithms before the actual front-end is really built. It could even be used to evaluate different tradeoffs without the need of complex and time consuming experimental setups.

\section{METHODS}

\section{A. Simulation Platform}

The analog front-end (crystal layers, PS-PMT, analog electronics and ADCs) and the digital signal processing algorithms have been modeled using Simulink 5.0 (The Mathworks, Natick, MA). Modeling of the processing algorithms has been realized with a HW implementation in mind, i.e., including details of their actual implementation in VHDL, like computation delays or fixed-point arithmetic.

Additionally these processing algorithms have been implemented as digital blocks described with synthesizable VHDL (Very High Speed Integrated Circuit Hardware Description Language) optimized for their implementation on programmable logic (FPGA) from Xilinx (Xilinx, San José CA, USA). Modelsim SE (Mentor Graphics, Wilsonville, USA) has been the 
VHDL simulator of choice. The software package XtremeDSP ${ }^{\circledR}$ from Xilinx has been used for the VHDL code verification and performance estimation. This package provides the coupling between Simulink and Modelsim, where the first provides the modeling and analysis while the latter accurately simulates the behavior of the hardware that will be synthesized. VHDL code evaluation and performance estimation are based on the following set-ups (test benches), which intensively exercise the different sub-modules:

- Short test with fixed stimuli, for visual analysis of internal waveforms of the VHDL modules versus their equivalent Simulink modules.

- Position estimation, which emulates a flood acquisition where a uniform source irradiates the detector surface. The simulations sweep along the emission energy.

- Timing estimation, which verifies the functionality of the timestamp generator. Events are generated with a known phase with respect to the sampling clock and the generated stamp is compared to the expected value.

- Energy estimation, which simulates a source irradiating a fixed position for a given phoswich layer.

- DOI estimation, which measures the energy in a delay window for events that hit a known layer at a random clock phase and position.

- Abnormal situations, the implemented HW may detect certain errors, such as saturations while integrating or unreliable time stamps, which may take place under certain conditions. This test forces the logic to such conditions.

\section{B. System Model}

Synthetic anode pulses are built by modeling the different stages of the acquisition chain, taking into account the statistical properties of the processes involved. The starting point is the average charge $\overline{Q_{o}}$ delivered by each anode of the photomultiplier, which may be computed as:

$$
\bar{Q}_{o}=\bar{N}_{\mathrm{PHO}} \cdot \eta \cdot \bar{G}
$$

where $\bar{N}_{\text {PHO }}$ is the mean number of photons created in the scintillator upon $\gamma$-absorption, $\eta$ represents the probability that an optical photon from the scintillator reaches the first dynode of the PS-PMT and G represents the mean electron multiplication factor of the PS-PMT [7]. This charge is delivered in the form of a current pulse, which after preprocessing can be acquired and analyzed by the digital electronics.

Crystal Model: On every $\gamma$-ray scintillator interaction there is an energy exchange which generates as a result a certain amount $N_{\mathrm{PHO}}$ of optical photons. The actual number of generated photons is function of the $\gamma$-ray energy $E_{\gamma}$, the scintillator photoelectric fraction $P_{\mathrm{FE}}$, the photon yield $Y\left(E_{\gamma}\right)$ and the scintillator intrinsic energy resolution $\Delta E / E$. To model the energy deposition $E_{\text {CRYSTAL }}$, it is assumed that there is a photoelectric deposition with probability $P_{\mathrm{FE}}$, and a Compton deposition with probability $1-P_{\mathrm{FE}}$. In case of a Compton interaction, the deposited energy is random value that depends on the actual energy of the $\gamma$-ray and the recoil energy of the photon [8].
Moreover these photons are time distributed according to the scintillation properties of the crystal, which can be modeled as a lineal combination of two or more exponential functions [9]. These functions take into account pulse rise $\tau_{\mathrm{RISE}}$ and fall $\tau_{\text {FALL }}$ constants. It has been shown that most scintillators of interest may be described with a single rise and a single fall constant [5]. Putting all together, the crystal pulse is modeled as follows:

$$
\begin{aligned}
E_{\mathrm{CRISTAL}} & = \begin{cases}E_{\gamma}=\mathrm{h} \nu & \text { with } \operatorname{Prob}\left(\mathrm{P}_{\mathrm{FE}}\right) \\
\mathrm{U}\left(0, \frac{E_{\gamma}^{2}}{E_{\gamma}+255}\right) & \text { with } \operatorname{Prob}\left(1-P_{\mathrm{FE}}\right)\end{cases} \\
N_{\mathrm{PHO}} & =N\left(\mathrm{E}_{\mathrm{CRISTAL}} \cdot \text { Yield }, \frac{\Delta E}{\mathrm{E}_{\mathrm{CRISTAL}}}\right) \\
N_{\mathrm{PHO}}(t) & =N_{\mathrm{PHO}} \frac{\exp \left(-t_{\tau_{\mathrm{FALL}}}\right)-\exp \left(-t_{\tau_{\mathrm{RISE}}}\right)}{\tau_{\mathrm{FALL}}-\tau_{\mathrm{RISE}}}
\end{aligned}
$$

where $U(a, b)$ stands for a Uniform distribution between $a$ and $b$, and $N(m, v)$ stands for a Gaussian distribution with mean $m$ and variance $v$.

The second term $\eta$ of (1) is decomposed as the product of two terms: crystal efficiency $\eta_{\mathrm{CRYS}}$ and quantum efficiency $\eta_{\mathrm{PMT}}$. The first term ranges between 20-60\% depending on crystal size, surface finish and coupling to the PMT [10], while the latter depends on PMT efficiency at the wavelengths defined by the scintillator emission spectrum and is usually less than $25 \%$. They are implemented as a binomial distribution, where the actual number of photons $N_{\mathrm{PHO}}^{\prime}(t)$ that reach the photocathode during a short time interval $\Delta t$ around $\mathrm{t}$ is:

$$
\begin{aligned}
& N_{\mathrm{PHO}}^{\prime}(t) \\
& \quad=\frac{1}{\Delta t} \arg n\left\{U(0,1)=P\left(n \mid N_{\mathrm{PHO}}(t)\right)\right\} \\
& P\left(n \mid N_{\mathrm{PHO}}(t)\right) \\
& \quad=\left(\begin{array}{c}
t+\Delta t / 2 \\
\int_{t-\Delta t / 2}^{t} N_{\mathrm{PHO}}(t) d t \\
P(
\end{array}\right) \cdot \eta^{n} \cdot(1-\eta)^{N_{\mathrm{PHO}}(t)-n} \approx \\
& \quad=\left(\begin{array}{c}
\left.N_{\mathrm{PHO}}(t)\right) \\
n
\end{array}\right) \cdot \eta^{n} \cdot(1-\eta)^{N_{\mathrm{PHO}}(t)-n}
\end{aligned}
$$

In case of a multi-layer phoswich, in first place the crystal layer is selected randomly, taking into account the a priori probabilities of each layer which are function of crystal lengths and the scintillator stopping power, and next a pulse is drawn out as described in previous $(2)(3)(4)(5)$.

PS-PMT Model: The next item in the chain is the PMT, whose basic functional diagram is shown in Fig. 2. It consists of a photocathode and a series of dynodes in an evacuated glass enclosure. When photons strike the photocathode, electrons are emitted via the photoelectric effect. These electrons are focused onto the first dynode by an electric field and when they strike the dynodes, more electrons are emitted. The current of electrons increases as it moves down the successive dynodes until it reaches the anode, producing a small current pulse, achieving 

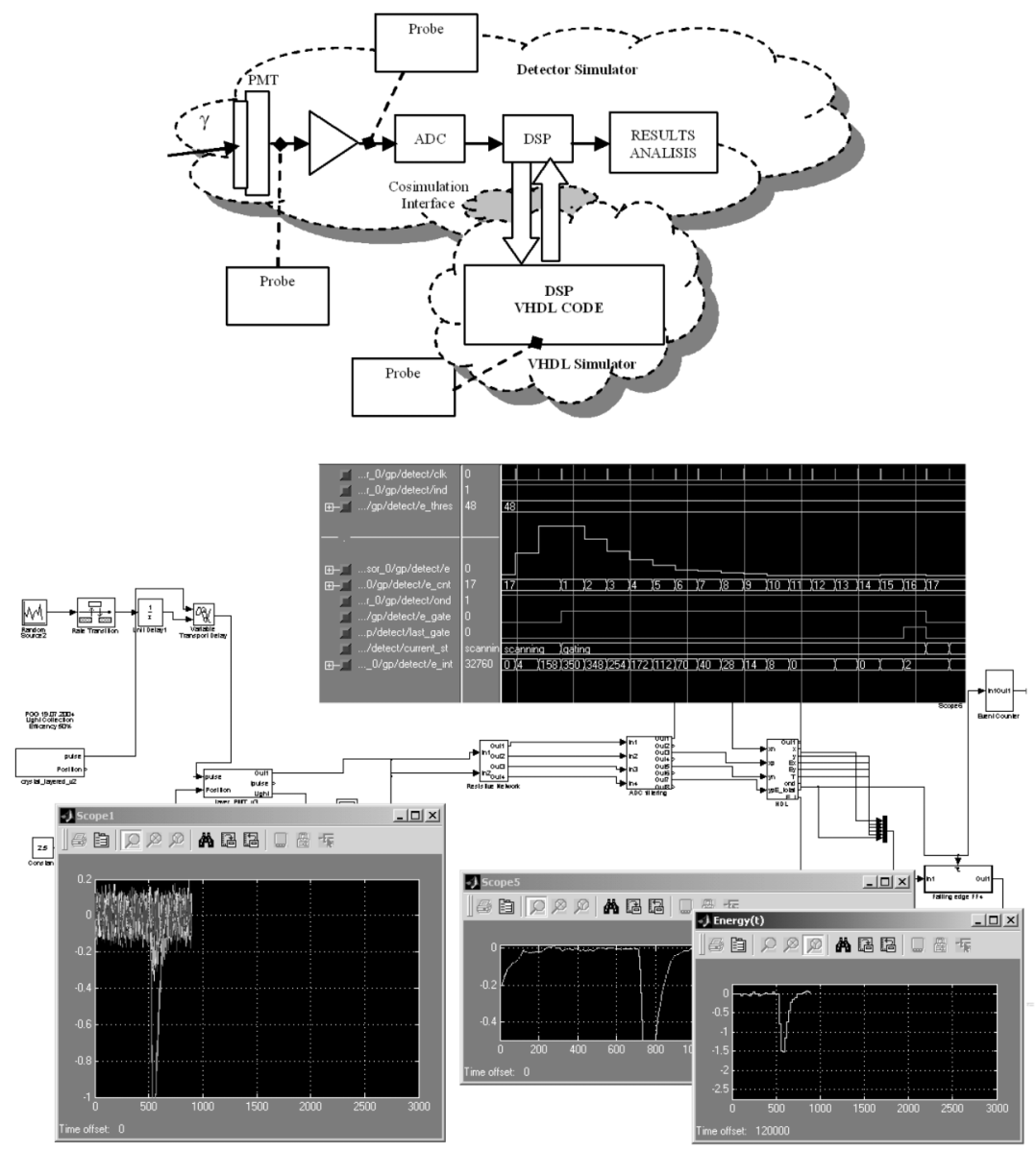

Fig. 1. The simulation platform, as it is shown it the upper figure, consists of two concurrent simulators. The first, which is based on Simulink, simulates the functional part of the system providing the input patterns to the Modelsim. The latter simulates the HW that is actually going to be synthesized and implemented. Both tools are linked through the cosimulation interface provided by System Generator from Xilinx. The lower figure shows a feasible set up, with the Simulink schematic in the background and probes outputs in the foreground. These probes include a VHDL waveform provided by Simulink and the outputs of the PS-PMT and the inputs to the ADCs. Such approach provides a complete picture of what is going on at each point of the system.

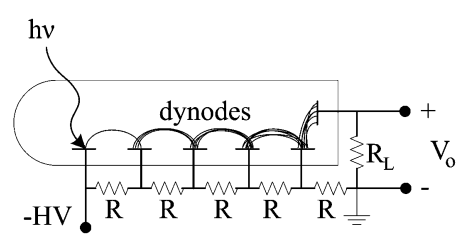

Fig. 2. Schematic of a basic PMT. A high voltage (HV) is applied over the photocathode, the dynodes and the anode to create an electric field that focuses electrons towards the collecting anode. This is done by the aid of a resistor chain (R) that creates a potential ladder down the structure.

an amplification gain of $10^{3}-10^{8}$. The voltage divider network shown in the figure carries a steady current $-\mathrm{HV} / R_{\text {tot }}$ which supplies the electrons for multiplication at each dynode.

PMT's use is widely spread mostly because of their high linearity and gain. The most important parameters are the gain, which is function of voltage and temperature, dark current, transit time, rise time as well as cross-talk and gain non-uniformities. The implemented PMT simulation model extends the statistical approach presented at [11] to a PS-PMT, where each cathode collects and amplifies the energy striking a particular section of the PMT surface.
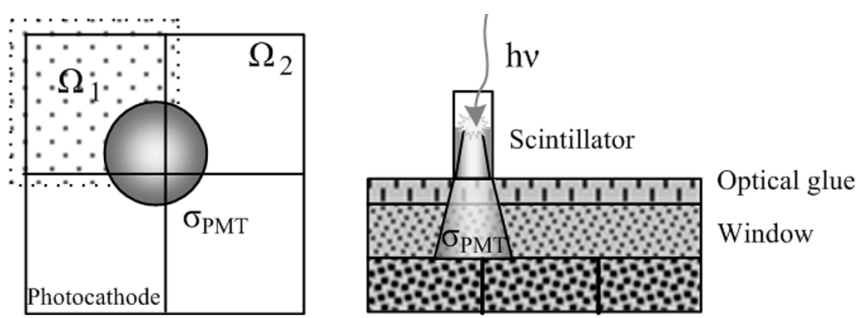

Fig. 3. The scintillation light pulse from a given crystal irradiates an area of the PMT surface, which includes several photocathodes. Each of these collects and amplifies the energy, giving away a current pulse through its anode that is function of the impact location and also of the PMT properties.

As it is shown in Fig. 3, the photons coming out the crystal illuminate an area of the photocathode bigger than the crystal section due to the photon spread during their propagation through the optical glue and borosilicate window. This spread has been modeled as a spatially distributed gaussian function centered at the crystal whose spread factor $\sigma_{\mathrm{PMT}}$ is empirically determined. In this way the energy collected by each anode $\mathrm{j}$ is computed as 
the integral across the corresponding cathode domain $\Omega_{j}$ as follows:

$$
\begin{array}{r}
F_{j}=\frac{G_{j}}{\sqrt{2 \pi \sigma_{\mathrm{PMT}}^{2}}} \int_{\Omega_{j}} \exp \left(-\frac{\left(x-x_{m}\right)^{2}+\left(y-y_{m}\right)^{2}}{2 \sigma_{\mathrm{PMT}}^{2}}\right) \\
\cdot d x \cdot d y
\end{array}
$$

where $\left(x_{m}, y_{m}\right)$ are the coordinates of the $m$ crystal center at which energy deposition takes place, $G_{j}$ includes gain non-uniformities and $\sigma_{\mathrm{PMT}}$ models photon spread.

Finally the single-photon PMT response, characterized by the device's rise time $t_{\mathrm{PMTrise}}^{2}$ and transition time jitter $t_{\mathrm{PMTjitter}}^{2}$, is modeled as:

$$
\begin{aligned}
h(t) & =\frac{1}{t_{p}^{2}} \cdot t \cdot \exp \left(-\frac{t}{t_{p}}\right) \\
t_{p}^{2} & =t_{\mathrm{PMTrise}}^{2}+t_{\mathrm{PMT} \text { jitter }}^{2}
\end{aligned}
$$

Combining all these expressions together we estimate the current $i_{j}(t)$ at the output of each anode $\mathrm{j}$ as the PMT response to the photon flux illuminating the cathode plus the PMT dark current $I_{\text {dark }}$ as follows, where G is the PMT gain and $\mathrm{q}$ is the charge of an electron:

$$
i_{j}(t)=q \cdot \bar{G} \cdot F_{j} \cdot h(t) * N_{\mathrm{PHO}}^{\prime}(t)+I_{\mathrm{dark}}
$$

Readout Model: Two options are considered for anode readout; on one side, the classical Anger approach where the number of processed signals is reduced by means of a resistive network [1], on the other each anode is individually acquired [12]. In any case current pulse is amplified by a transimpedance filter and shaped by a $\mathrm{CR}-\mathrm{RC}^{n}$ filter [13] prior to digital conversion by an analog-to-digital converted (ADC) sampling at a fix rate $f_{s}$. The acquired stream will be digitally processed to identify and characterize the pulses generated by the detected $\gamma$-ray.

At this point it is required to stress the considered noise model, as it is essential to achieve a realistic synthetic pulse. The implemented model considers both series $e_{n}^{2}$ and parallel $i_{n}^{2}$ noise according to the noise model described in Fig. 4. It has been shown [14] that under normal conditions the noise spectral density may be modeled as a first polynomial, where the coefficients $a, b, a_{f}, b_{f}$ are either analytically estimated or empirically computed:

$$
\begin{aligned}
& \frac{d e_{n}^{2}}{d f}=a+\frac{a_{f}}{|f|} \\
& \frac{d i_{n}^{2}}{d f}=b+b_{f}|f|
\end{aligned}
$$

\section{RESULTS}

The presented models for the analog front-end have been implemented as parametrizable Simulink blocks, which are easily configured to simulate different scenarios or test benches.

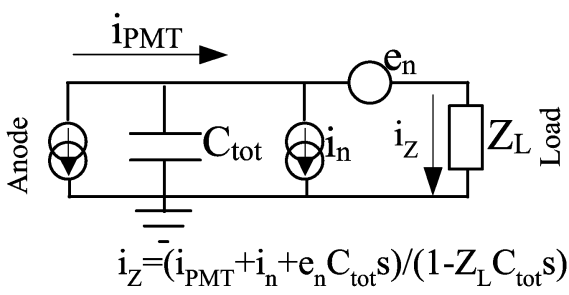

Fig. 4. Noise model for the analog front-end, which considers two noise source. series and parallel noise. It is important to point out the influence of parasitic capacitances $\mathrm{C}_{\text {tot }}$ on the series noise which may dominate over the rest of noise sources.

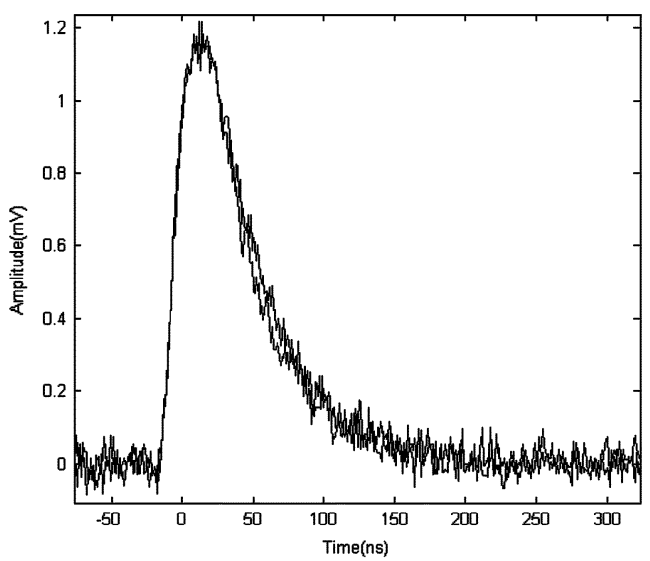

Fig. 5. Synthetic (light gray) scintillation pulse versus real (black) pulse for a LYSO crystal (Crystal Photonics Inc).

TABLE I

Estimated VALUES FOR THE SERIES AND PARALlel White NOISE BASED ON THE ANALYTICAL EXPRESSIONS OF [14] AND MEASURED DATA. As A FIRST APPROXIMATION IT IS CONSIDERED THAT WHITE NOISE DOMINATES OVER 1/F NOISE

\begin{tabular}{|c|c|c|}
\hline Series white noise & Parallel white noise & Equivalent leakage current \\
\hline $200 \mathrm{nV} / \mathrm{Hz}^{1 / 2}$ & $14 \mathrm{fA} / \mathrm{Hz}^{1 / 2}$ & $126 \mathrm{nA}$ \\
\hline
\end{tabular}

The first of these scenarios mimics an available LYSO-based detector whose pulses are compared, as shown in Fig. 5, to real dynode pulses acquired with an oscilloscope. As it can be seen, both shape and noise level are identical.

The next stage has been the configuration of the Simulink blocks to model an analog front-end consisting of a $16 \times 16$ $\operatorname{LSO}(0.22 \times 0.22 \times 1.2 \quad \mathrm{~cm}) / \mathrm{GSO}(0.22 \times 0.22 \times 1.2 \quad \mathrm{~cm})$ phoswich coupled to a Hamamatsu H5000 PS-PMT [15], with optical grease, based on the observations and measurements carried out by several authors [3], [6], [7], [12], [16]-[18]. Given the scintillator, coupling and PMT properties the overall efficiency $\eta$ is set to $10 \%$ which is consistent with previously reported values [7], [19]. Noise levels, whose values are shown in Table I, are based on the measurements done with our current front-end. Anode outputs are reduced to 4 Anger signals namely $(\mathrm{X}+, \mathrm{X}-, \mathrm{Y}+, \mathrm{Y}-)$ by means of a resistive network [1], each of which is filtered by a CR-RC filter with 10 ns peaking time and continuously sampled at $50 \mathrm{MHz}$ by a 10 bit ADC. Finally, the original PMT has been simplified from $8 \times 8$ to $4 \times 4$ channels in order to speed-up simulations and provide a pessimistic scenario for performance estimations. 

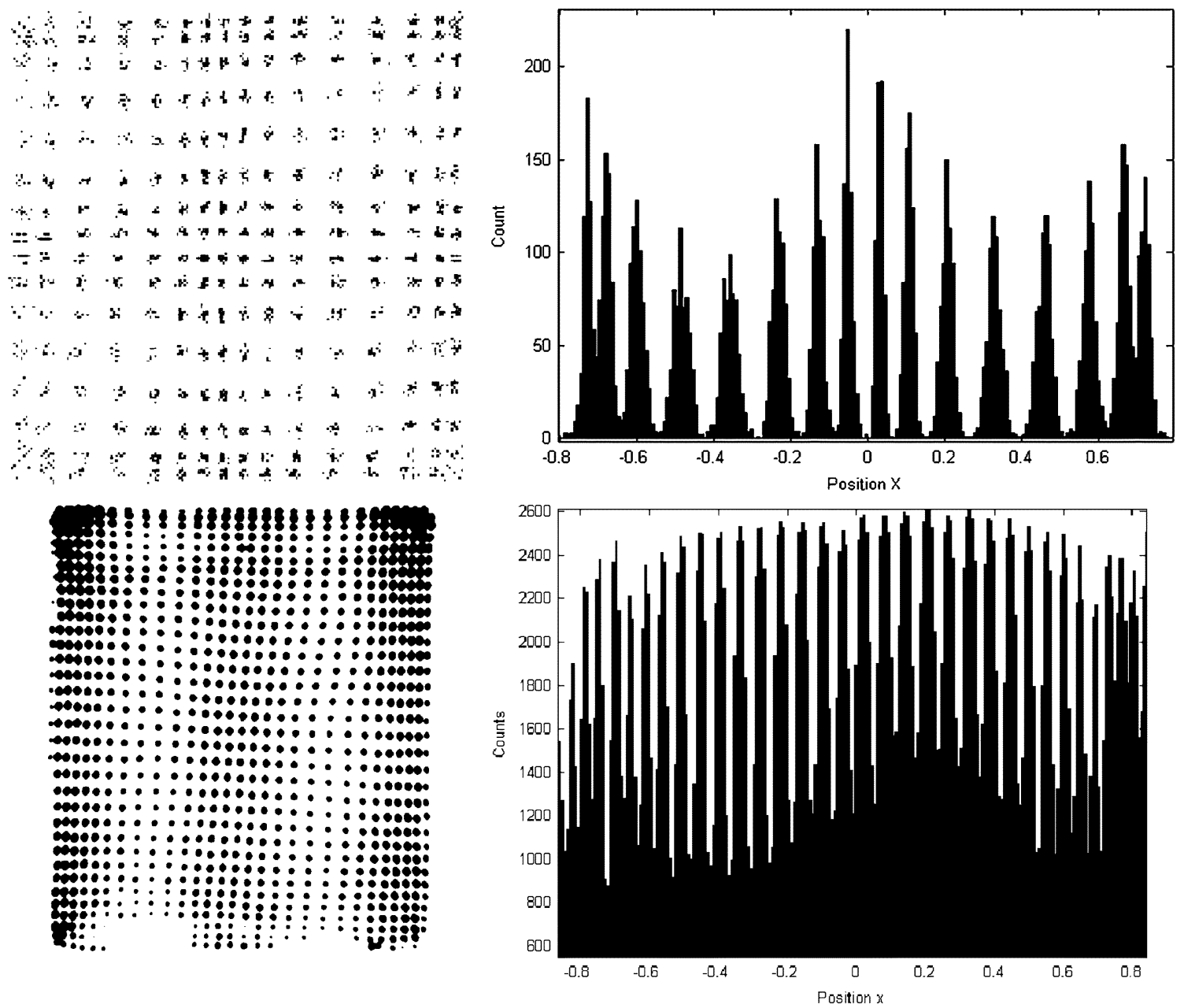

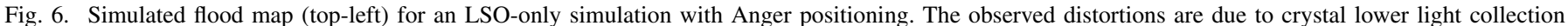

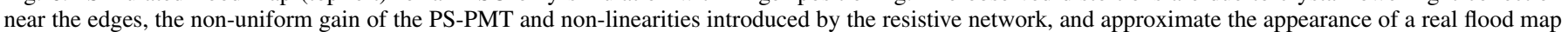

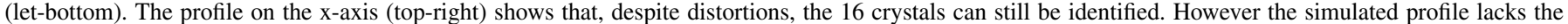
background noise appreciated in a real profile (bottom-right).

TABLE II

BRIEF COMPARISON BETwEEN SIMULATIONS AND PUBLISHED RESUlTS

\begin{tabular}{|l|l|c|l|}
\hline & Habte et al[3] & Pani et al[18] & Simulations \\
\hline Gain & $1: 2.2$ & -- & $1: 3$ \\
\hline $\begin{array}{l}\text { Peak-Valley } \\
\text { Ratio }\end{array}$ & $13: 1$ & $21: 1$ & $15: 1$ \\
\hline Cross-talk & $5 \%$ & $3.22 \%(5 \mathrm{~mm})$ & $4.5 \%$ \\
\hline$\Delta \mathrm{E} / \mathrm{E}$ & $25 \%$ & -- & $18.8 \%$ \\
\hline
\end{tabular}

Regarding the ADCs models, these included parameters for distortions and non-linearities. However in order to speed up simulations, these effects were exchanged for a reduced ADC dynamic range, in such a way that only the lower 8.5 bits are really used. The data stream generated by the ADCs is used as input stimuli to the VHDL code which is concurrently simulated by Modelsim. These stimuli have been used to debug and validate the implemented digital algorithms: pulse detection, energy, position, time stamp generation and pulse shape for DOI estimation.

Fig. 6 shows a flood map, both simulated and real for a LSOonly detector. Distortions on the crystal location may be attributed to the crystals lower light collection near the edges, the non-uniform gain of the PS-PMT and non-linearities introduced

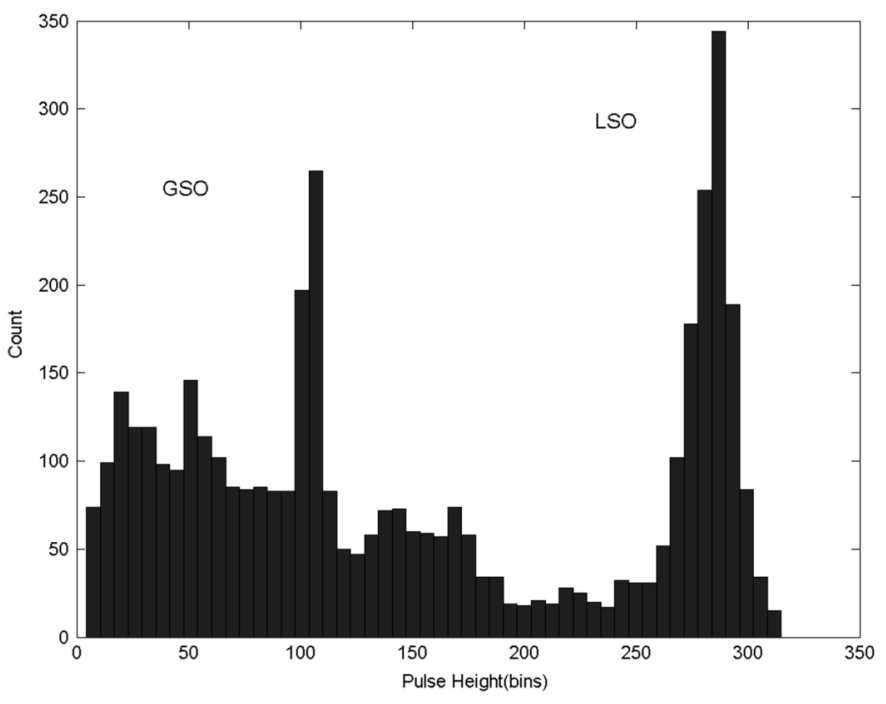

Fig. 7. Simulated energy spectrum for a fix position of the crystal map, it is observed that GSO and LSO $511 \mathrm{KeV}$ photopeaks are clearly identified. The FHWM energy resolution is estimated to be around $15.5 \%$ for GSO and $11.5 \%$ for LSO. The relative gain between LSO and GSO is 2.7:1, as expected due to the lower GSO photon yield and a slightly lower PS-PMT quantum efficiency at the GSO wavelength. 


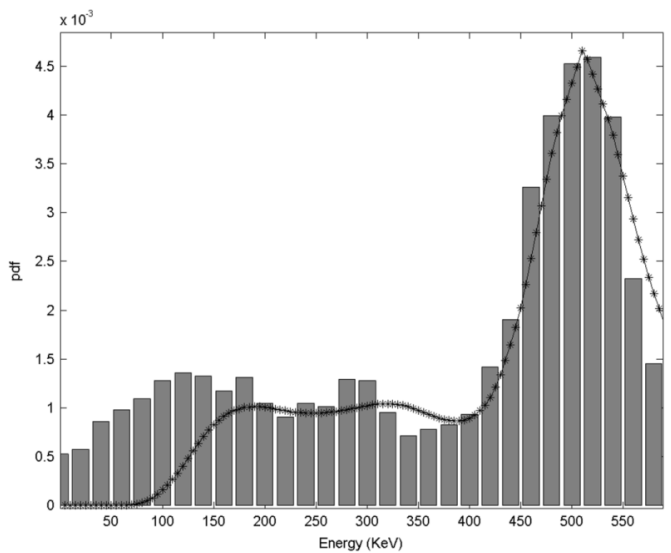

Fig. 8. Simulated energy spectrum (bars) versus measured spectrum (stars) for a LYSO crystal. Data is normalized to represent a probability density function between $0 \mathrm{a} 600 \mathrm{KeV}$. The difference in the low energy band is due to a higher triggering threshold of the real system.

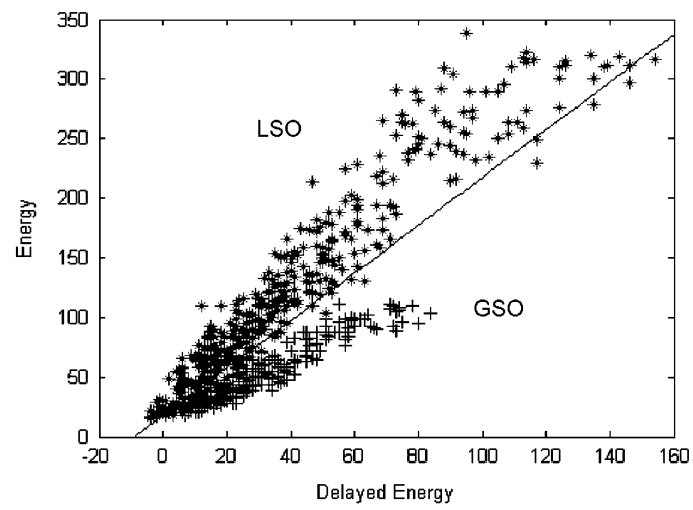

Fig. 9. Simulated phoswich diagram for LSO and GSO. The y-axis is the result of integrating the complete pulse energy while in the X-axis only a few samples of the pulse tail are integrated. Due to HW implementation both energies are related with a scale factor of 4 .

by the resistive network and, as it is observed in the figure, the simulation model is able to reproduce these effects.

Event position (Fig. 6), energy (Fig. 7) and Table II summarizes a brief comparison between theses simulations and published measurements with similar setups.

\section{CONCLUSION AND FUTURE WORK}

Our simulation results show very good agreement with published results for similar configurations [3], [18] and suggest that this relatively simple system models are able to describe the most significant system features and provide realistic stimuli to the VHDL, fact that eases the implementation and validation of the implemented algorithms on hardware as well as enables the estimation of system performance. As an example, the availability of synthetic stimuli gave valuable insight for the design and optimization of the digital timestamp generation block and allowed for the detection of a very subtle code bug which would have been very difficult otherwise. However computation time may be an issue when it comes to performance estimation: it takes around 7 hours on a Pentium 4 running at $3.2 \mathrm{GHz}$ to simulate 100000 pulses which is enough for code validation but may not be sufficient for an accurate performance estimation.
The implemented blocks estimate the most significant parameters of the pulse: energy, position, timestamp and DOI, assuming a continuous data stream generated by free running ADC. As future work, we are currently working on the elaboration of a prototype where pulse will be acquired up to $65 \mathrm{MHz}$, characterized by the processing logic and sent to the host via a Ethernet connection. Additionally we are working on a new VHDL code that takes as input each anode output so that we get rid of the resistive network, and the presented cosimulation environment will be extensively used for debugging, validation and performance estimation.

\section{REFERENCES}

[1] S. Siegel, R. W. Silverman, Y. Shao, and S. R. Cherry, "Simple charge division readouts for imaging scintillator arrays using a multi-channel PMT," IEEE Trans. Nucl. Sci., vol. 43, pp. 1634-1641, 1996.

[2] V. Popov, S. Majewski, A. G. Weisenberger, and R. Wojcik, "Analog readout system with charge division type output," in IEEE Nucl. Sci. Symp., 2001, vol. 4, pp. 1937-1940.

[3] F. Habte, P. D. Olcott, C. S. Levin, A. M. Foundray, and J. A. Talcott, "Prototype parallel readout system for position sensitive PMT based gamma ray imaging systems," in IEEE Nucl. Sci. Symp., Portland, OR, 2003, vol. 3, pp. 1891-1894.

[4] J. Seidel, J. J. Vaquero, and M. V. Green, "Resolution uniformity and sensitivity of the NIH ATLAS small animal PET scanner: Comparison to simulated LSO scanners without depth-of-interaction capability," IEEE Trans. Nucl. Sci., vol. 50, pp. 1347-1350, 2003.

[5] A. Thon, K. Fiedler, T. Frach, W. Rütten, and T. Solf, "Exact modeling of analog pulse for detector modules," in IEEE Nucl. Sci. Symp., Portland, OR, 2003, vol. 4, pp. 2227-2231.

[6] J. Seidel, J. J. Vaquero, S. Siegel, W. R. Gandler, and M. V. Green, "Depth identification accuracy of a three layer phoswich PET detector module," IEEE Trans. Nucl. Sci., vol. 46, pp. 485-490, 1999.

[7] P. Dorenbos, J. T. M. de Haas, and C. W. E. van Eijk, "Non-proportionality in the scintillation response and the energy resolution obtainable with scintillation crystals," IEEE Trans. Nucl. Sci., vol. 42, pp. 2190-2202, 1995.

[8] J. A. Sorenson and M. E. Phelps, Physics in Nuclear Medicine, Second ed. Orlando, FL, USA: Harcourt Brace Jovanovich, 1987.

[9] M. e. Ljungberg and M. A. e. King, Monte Carlo Calculations in $\mathrm{Nu}$ clear Medicine: Applications in Diagnostic Imaging. Philadelphia, PA: Institute of Physics Publishing, 1998.

[10] C. S. Levin and F. Habte, "Initial studies of a new detector design for ultra-high resolution positron emission tomography," in IEEE Nucl. Sci. Symp., 2002, vol. 3, pp. 1751-1755.

[11] Burle, Photomultiplier Handbook. Lancaster, PA: Burle Industries, 1989.

[12] S. Riboldi, "Investigation of signal readout methods for the hamamatsu R8500 flat panel PSPMT," in IEEE Nucl. Sci. Symp., Portland, OR, 2003, vol. 4, pp. 2452-2456.

[13] J. Wulleman, "Detector-noise suppression by appropriate CR-(RC)n shaping," Electron. Lett., vol. 32, pp. 1953-1954, 1996.

[14] G. Bertuccio and A. Pullia, "A method for the determination of the noise parameters in preamplifying systems for semiconductor radiation detectors," Rev. Scientific Instruments, vol. 64, pp. 3294-3298, 1993.

[15] H8500 Datasheet, , 2003 [Online]. Available: http://sales.hamamatsu. com/index.php?id=13195715, Hamamatsu

[16] M. Moszynski, T. Ludziejewski, D. Wolski, W. Klamra, and V. V. Avdejchikov, "Timing properties of GSO, LSO and other Ce doped scintillators," Nuclear Instruments and Methods in Physics Research Section A, vol. 372, pp. 51-58, 1996.

[17] R. Pani, "Factors affecting flat panel PMT calibration for gamma ray imaging," in IEEE Nucl. Sci. Symp., 2002, vol. 2, pp. 671-675.

[18] R. Pani, "Recent advances and future perspectives of position sensitive PMT," Nuclear Instruments and Methods in Physics Research Section A, vol. 213, pp. 197-205, 2004.

[19] W. W. Moses and S. E. Derenzo, "Prospects for time-of-flight PET using LSO scintillator," IEEE Trans. Nucl. Sci., vol. 46, pp. 474-478, 1999.

[20] M. B. Streun, G. Larue, H. Saleh, H. Zimmermann, E. Ziemons, K. Halling, and H. , "Pulse shape discrimination of LSO and LuYAP scintillators for depth of interaction detection in PET," IEEE Trans. Nucl. Sci., vol. 50, pp. 344-347, 2003.

[21] E. Geraniotis and H. Poor, "Robust matched filters for optical receivers," IEEE Trans. Commun., vol. 35, pp. 1289-1296, 1987. 